\title{
Analytical kinetics of clustering processes with cooperative action of aggregation and fragmentation
}

\author{
Vladimir M. Dubovik, ${ }^{1}$ Arkadi G. Galperin, ${ }^{2}$ and Viktor S. Richvitsky ${ }^{3}$ \\ (Joint Institute for Nuclear Research (JINR), Dubna, Moscow reg., 141980, Russia) ${ }^{4}$ \\ Aleksey A. Lushnikov ${ }^{4}$

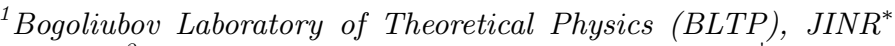 \\ ${ }^{2}$ Laboratory of High Energies (LHE), JINR \\ ${ }^{3}$ Laboratory of Information Technologies (LIT), JINR \\ 4 Karpov Institute of Physical Chemistry (NIFHI), Moscou
}

(Dated: October 25, 2018)

\begin{abstract}
Some models of clustering processes are formulated and analytically solved employing generating functions methods. Those models include events which result from combined action of the coagulation and fragmentation processes. Fragmentation processes of two kinds, so-called similar and arbitrary, ones, are brought forward, and the explicit forms of their solutions are produced. This implies some possibility of existence of different aggregation mechanisms for clusters creation differing in their inner structure. All the models are based on "the three-level bunch" scheme of interaction between the system states. Those states are described in terms of the probability to find the system in the state with an exactly given number of clusters. The models are linear in the probability functions due to the assumption that the rates of elementary acts are permanent. Some peculiarities of application of the generating function method to solution of the linear differential-difference equations are revealed. The illustration of the problem in terms of a traffic jam picture is not a specific one.

PACS numbers: 02.50.-r, 89.40.+k

Keywords: cluster, clustering, aggregation, coagulation, fragmentation, decay, stochastic, differentialdifference equation, generating function
\end{abstract}

\section{INTRODUCTION}

In the present work we develop and define more accurately our earlier considerations [1]. A cluster is generally understood as either a number of things of the same kind growing together or number of particles, objects, etc., in a small, close group. This idea is a very general and not a specified one. An aggregation process in the form $a u+b u=(a+b) u$ is referred as coagulation, when coefficients $a, b$ represent the quantities of the scale unit of $u$ which are coalesced with time. Aggregation and fragmentation are a couple of mutually inverse processes. The physical scales ( spatial, temporal, value of masses, e.t.c.) vary in the many orders of a magnitude for such a processes. That is why, an idea of existence of a universal description of the above phenomena arises and the unification of both direct and inverse processes in a general class of clustering processes seems being of a natural one.

The clustering processes resemble to a certain degree the nucleation processes. This roots in the mathematical description being general for kinetics of such processes

\footnotetext{
*Electronic address: dubovik@thsun1.jinr.ru

${ }^{\dagger}$ Electronic address: galperin@vxjinr.jinr.ru

${ }^{\ddagger}$ Electronic address: gavtsk@cv.jinr.ru

$\S$ Electronic address: lalush@cc.nifhi.ac.ru
}

despite the lack of obvious resemblance in their actual more precise details. On the one hand, we can see those approaches in the course of investigations on the molecular and submolecular level, in theories of condensed matter, nuclei and nuclear chains 22. On the other hand, clustering of disperse systems are considered in astrophysics (forming of cosmic objects), atmospheric science, chemistry, ... [3].

For example, an expanding universe is formed not at once. Clusters grow by coalescence of smaller clusters. Their growth kinetics is like to the kinetics of coagulation. In what follows we formulate basic equations and outline the methods for their solution. Moreover, one could expect that theoretical tools developed to describe physical systems can be exploited in other fields, such as ecology of computation [4] or biology, economics, transport problem, etc. [5, 6].

Consider the kinetics of formation of a $G$-cluster using the picture of a one-way motor lane. We assume that the starting configuration is $G$ independent cars on the motor lane, the leading one being the slowest, and no one can pass over each other. So, each initial cluster contains one car.

The process begins at $t=0$. On passing some time $t$, the initial $G$ cars group in clusters containing $g_{1}, g_{2} \ldots g_{s}$ cars. These clusters go on to coalesce. The problem is to determine the time evolution of the probability $w_{s}\left(g_{1}, g_{2} \ldots g_{s} ; t\right)$ to find $s$ clusters $g_{1}, g_{2} \ldots g_{s}$. The sum of their masses $g_{k}$ are subjected to the constraint (con- 
servation law):

$$
\sum_{k=1}^{s} g_{k}=G
$$

in the system. Thus, we concern only of non-relativistic events and study systems with a permanent (additive) mass and a finite number of particles.

Our goal is to formulate and investigate the exactly solved models of the clustering (dissociation) processes, including those which result from combined action of a certain aggregation and fragmentation.

The paper is organized as follows. In Section II, we explain the stochastic motion of our objects, obtain the probability of finding out the system in the state of exactly $s$ clusters and dependent on the time average number of clusters by means of introducing a generating function. Later on, we formulate a master equation governing the time evolution of the probability of finding the clusters of various masses (Sect. III). We solve that problem applying the Laplace transformation with respect to $t$ to master equation (Sect. IV). Then we find the probability to detect a cluster of assigned mass $g$ by summation of $w_{s}$ over all $g_{k}$ irrespective of the distribution of other participants, except for the selected one (Sect. V). Some properties of those convolutions are true due to the isomorphism between a set of generating functions with a product operation and a set of $w(g)$ with convolution (see Appendix as well), which have the structures of semigroups with unit. In the following we consider processes of similar fragmentation (Sect. VI), combined action of aggregation and similar fragmentation (Sect. VII), and the process with an arbitrary fragmentation (Sect. VIII). In conclusion, we discuss our results.

\section{NUMBER OF CLUSTERS: PURE AGGREGATION PROCESS}

Let $\gamma$ be the rate of an elementary coalescence act; two adjacent clusters produce a single one (for instance, a dimer is formed when a car catches up another one). We assume that $\gamma$ is $g$-independent. Then we can characterize the situation by the number of intervals between adjacent clusters. If there are $s$ clusters in the system, the number of intervals is $s-1$. Each coalescence act annihilates one interval. The number of ways to do this is exactly equal to the number of intervals.

Let $W(s, t)$ be the probability of meeting exactly $s$ clusters at the time $t$. Then:

$$
\frac{d W(s, t)}{d t}=\gamma[s W(s+1, t)-(s-1) W(s, t)] .
$$

One can observe a "three-level bunch" scheme of transitions between the three nearest (on $s$ ) states of the system under consideration. Equation (2) should be supplemented with the initial conditions:

$$
W(s, 0)=W_{0}(s) .
$$

In particular, if initially there were exactly $G$ independent cars, the function $W_{0}(s)$ obeys the equation

$$
W_{0}(s)=\Delta(s-G),
$$

with $\Delta$ being the Kronecker delta: $\Delta(0)=1$, and $\Delta=0$ otherwise.

Equation (2) can be solved by introducing the generating function:

$$
F(z, t)=\sum_{s} W(s, t) z^{s-1} .
$$

Combining Eqs. (21) and (5) gives:

$$
\partial_{t} F=(1-z) \partial_{z} F
$$

The rate $\gamma$ is included into the definition of time. The initial condition for an initially monodisperse system is rewritten in terms of $z$ as:

$$
F(z, 0)=z^{G-1} .
$$

The solution of Eq. (6) with the initial condition, Eq. (7), has the form:

$$
F(z, t)=\left[1-e^{-t}(1-z)\right]^{G-1} .
$$

The probability $W(s, t)$ is thus expressed in terms of binomial distributions:

$$
W(s, t)=C_{G-1}^{s-1} e^{-(s-1) t}\left(1-e^{-t}\right)^{G-s} .
$$

It is no problem to find the time dependence of the average number of clusters:

$$
\bar{s}(t)=\left.\partial_{z} z F(z, t)\right|_{z=1}=1+(G-1) e^{-t} .
$$

\section{MASS DISTRIBUTION IN A PURE AGGREGATION PROCESS}

In analogy with the kinetics of disperse systems, we shall refer to $g_{k}$ as the cluster mass. Our goal now is to formulate the master equation governing the time evolution of the probability $w_{s}\left(g_{1}, g_{2} \ldots g_{s} ; t\right)$ to find the clusters of masses $g_{1}, g_{2} \ldots$ at the time $t$. This equation is formulated as follows:

$$
\begin{aligned}
& \frac{d w_{s}}{d t}=\sum_{\left[g^{\prime}\right], k} w_{s+1}\left(g_{1} \ldots g_{k}^{\prime}, g_{k+1}^{\prime}, g_{k+2}^{\prime} \ldots g_{s+1}^{\prime}\right) \\
& \times \Delta\left(g_{k}-g_{k}^{\prime}-g_{k+1}^{\prime}\right) \\
& \times \Delta\left(g_{k+2}^{\prime}-g_{k+1}\right) \ldots \Delta\left(g_{s+1}^{\prime}-g_{s}\right)-(s-1) w_{s} .
\end{aligned}
$$

The meaning of the terms in the r.h.s. of Eq. (11) is rather apparent. The rate of losses is simply proportional to the number of empty intervals (the rate constant $\gamma$ is included into the definition of time). The gain occurs each time when two clusters of masses $g_{k}^{\prime}$ and $g_{k+1}^{\prime}$ coalesce producing a new cluster of mass $g_{k}$. Other $\Delta$-s simply restore the serial numbers of $g_{i}$ clusters with $i<k$ for the system of $s$ clusters. 
Of course, initial conditions to Eq. (11) should also be specified. We again assume that initially there were $\mathrm{G}$ separate cars:

$$
w_{G}(1,1 \ldots 1, t=0)=1 \text {, }
$$

and all other probabilities are 0 .

\section{SOLUTION TO BASIC EQUATION}

On applying the Laplace transformation with respect to $t$ gives, instead of Eq. (11),

$$
\begin{gathered}
=\sum_{\left[g^{\prime}\right], k} \bar{w}_{s+1}\left(g_{1} \ldots g_{k}^{\prime}, g_{k+1}^{\prime}, g_{k+2}^{\prime} \ldots g_{s+1}^{\prime}\right) \\
\times \Delta\left(g_{k}-g_{k}^{\prime}-g_{k+1}^{\prime}\right) \\
\times \Delta\left(g_{k+2}^{\prime}-g_{k+1}\right) \ldots \Delta\left(g_{s+1}^{\prime}-g_{s}\right),
\end{gathered}
$$

where barred $\bar{w}$ stands for the Laplace transform of $w\left(g_{1}, g_{2} \ldots ; t\right)$. The last equation of this set is readily solved (Eq. (9)) to yield:

$$
\bar{w}_{G}=\frac{1}{p+G-1} .
$$

Now let us try to seek for a solution to Eq. (11) in the form:

$$
\bar{w}_{s}\left(g_{1}, g_{2} \ldots ;, p\right)=\frac{A_{s}\left(g_{1}, g_{2} \ldots\right)}{(p+G-1)(p+G-2) \ldots(p+s-1)},
$$

where the coefficients $A$ are independent of $p$ and satisfy the following set of recurrence relations:

$$
\begin{gathered}
A_{s}\left(g_{1}, g_{2} \ldots\right) \\
=\sum_{\left[g^{\prime}\right], k} A_{s+1}\left(g_{1} \ldots g_{k}^{\prime}, g_{k+1}^{\prime}, g_{k+2}^{\prime} \ldots g_{s+1}^{\prime}\right) \\
\times \Delta\left(g_{k}-g_{k}^{\prime}-g_{k+1}^{\prime}\right) \\
\times \Delta\left(g_{k+2}^{\prime}-g_{k+1}\right) \ldots \Delta\left(g_{s+1}^{\prime}-g_{s}\right) .
\end{gathered}
$$

A useful sum rule

$$
Q_{s}=s Q_{s+1},
$$

follows immediately from Eq. (16), where $Q_{s}=$ $\sum A_{s}\left(g_{1}, g_{2} \ldots g_{s}\right)$ (summation runs over all $g$ ), or

$$
Q_{s}=\frac{(G-1) !}{(s-1) !}
$$

In fact, the expression $A_{s}\left(g_{1}, \ldots, g_{s}\right)$ depends on $s$ only. It does not depend on the distribution of numbers $g_{1}, \ldots, g_{s}$ provided that $\sum_{k=1}^{s} g_{k}=G, \quad g_{k} \geq 1$ are conserved.

It can be seen from Eqs. (11), (13), (9), and (2) that the problem under consideration splits into two subproblems. The first one is the time evolution problem. It deals with transitions between different states of the system and connects each three nearest adjacent states. The second subproblem is to scrutinize mass spectra. It is a pure combinatorial task. In fact, we have to deal with some population dynamics. Mass spectra at instant $t$ originate from the interchange of generations at a given $G$, and the proper weights depend on the whole set of possible transitions from $s+1$-states to the $s$-state under consideration.

With the induction method, one obtains that:

- $A_{G}(1, \ldots, 1)=1$ is the only possible value;

- the number of terms in formula Eq. (16) is equal to

$$
\sum_{k=1}^{s} g_{k}-1=\sum_{k=1}^{s} g_{k}-\sum_{k=1}^{s} 1=G-s
$$

for each $s$ fixed and $k$ fixed. Under the inductive assumption, one can write down $A_{s+1}\left(g_{1}^{\prime}, \ldots, g_{s+1}^{\prime}\right)=A_{s+1}$. From this it follows that $A_{s}\left(g_{1}, \ldots, g_{s}\right)=(G-s) A_{s+1}$ irrespective of a specific distribution of $g_{1}, \ldots, g_{s}$.

The recurrence equations obtained just now

$$
\begin{gathered}
A_{s}=(G-s) A_{s+1}, \\
A_{G}=1
\end{gathered}
$$

have solutions

$$
A_{s}=(G-s) !
$$

The time dependence can be readily restored by using the inversion

$$
\begin{gathered}
\frac{1}{(p+s-1)(p+s) \ldots(p+G-1)} \longrightarrow \\
\longrightarrow \frac{1}{(G-s) !} e^{-(s-1) t}\left(1-e^{-t}\right)^{G-s} .
\end{gathered}
$$

Equations (16), (17), and (21) reproduce Eq. (9) as well.

The final result is formulated as follows:

$$
\begin{gathered}
w_{s}\left(g_{1}, g_{2}, \ldots g_{s} ; t\right)=e^{-(s-1) t}\left(1-e^{-t}\right)^{G-s} \\
\times \Delta\left(G-g_{1}-g_{2}-\ldots g_{s}\right) .
\end{gathered}
$$

\section{SINGLE-CLUSTER DISTRIBUTION IN A PURE AGGREGATION PROCESS}

Here we determine the probability to find a cluster of mass $g$ irrespective of the distribution of other participants. To this end we sum $w_{s}$ over all $g_{k}$ except one $\left(g_{1}\right.$, for example):

$$
\begin{gathered}
w(g, t)=\sum_{g_{k}} w_{s}\left(g, g_{2}, \ldots g_{s} ; t\right) \\
=e^{-(s-1) t}\left(1-e^{-t}\right)^{G-s} \\
\times \sum_{g_{k}} \Delta\left(G-g-g_{2}-\ldots g_{s}\right) .
\end{gathered}
$$

Using the identities

$$
\begin{gathered}
\Delta(q)=\left\{\begin{array}{l}
1, q=0 \\
0, q=1,2, \ldots
\end{array}\right\}=\frac{1}{2 \pi i} \oint \frac{d z}{z^{q} z}, \\
\left\{\begin{array}{l}
0, q=0 \\
1, q=1,2, \ldots
\end{array}\right\}=\frac{1}{2 \pi i} \oint \frac{z d z}{z^{q}(1-z)}, \\
\frac{1}{2 \pi i} \oint \frac{d z}{z^{r+1}(1-z)^{R+1}}=C_{R+r}^{r}=C_{R+r}^{R},
\end{gathered}
$$




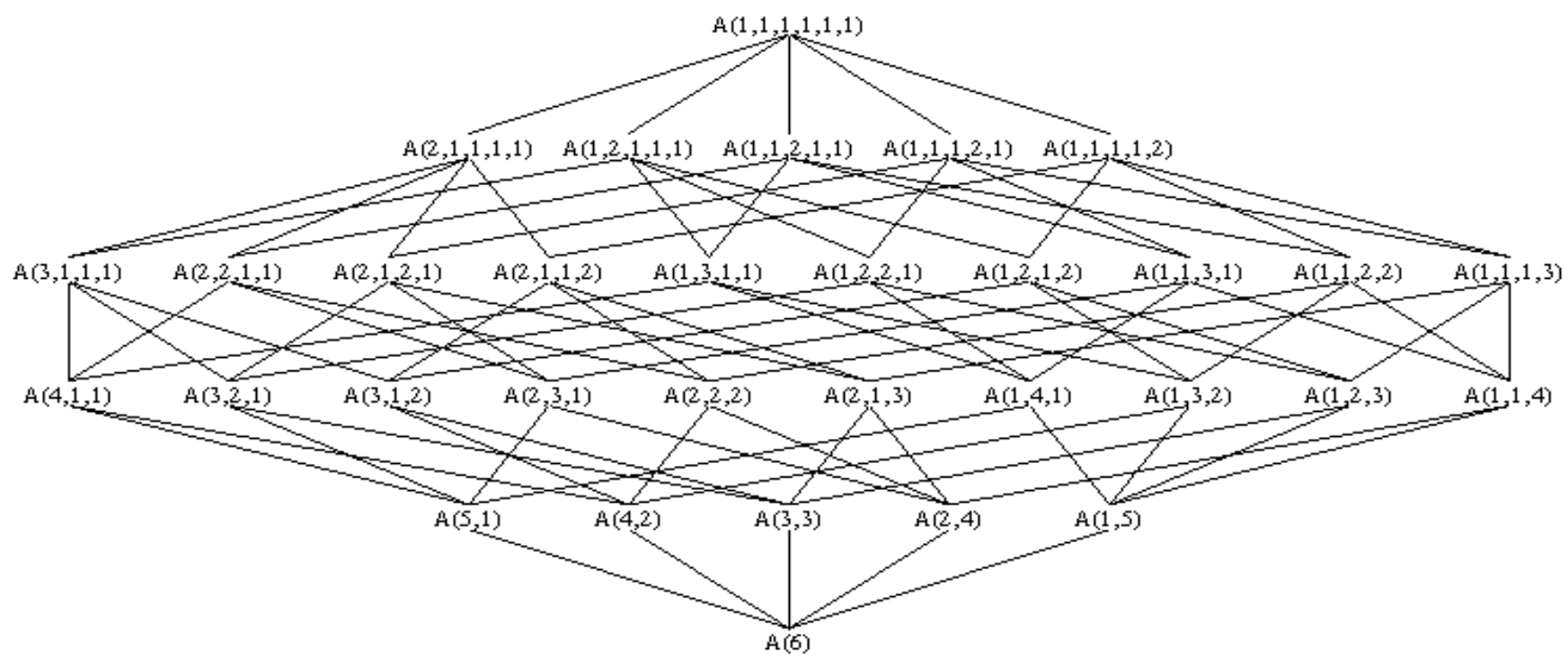

FIG. 1: Generation scheme for $\mathrm{G}=6$

one finds the convolution in Eq. (23):

$$
\begin{gathered}
w(g, t)=e^{-(s-1) t}\left(1-e^{-t}\right)^{G-s} \\
\times \frac{1}{2 \pi i} \oint \frac{z^{s-1} d z}{z^{G-g}(1-z)^{s-1} z} \\
=C_{G-g-1}^{s-2} e^{-(s-1) t}\left(1-e^{-t}\right)^{G-s} .
\end{gathered}
$$

Some important properties of the convolutions are discussed in Appendix.

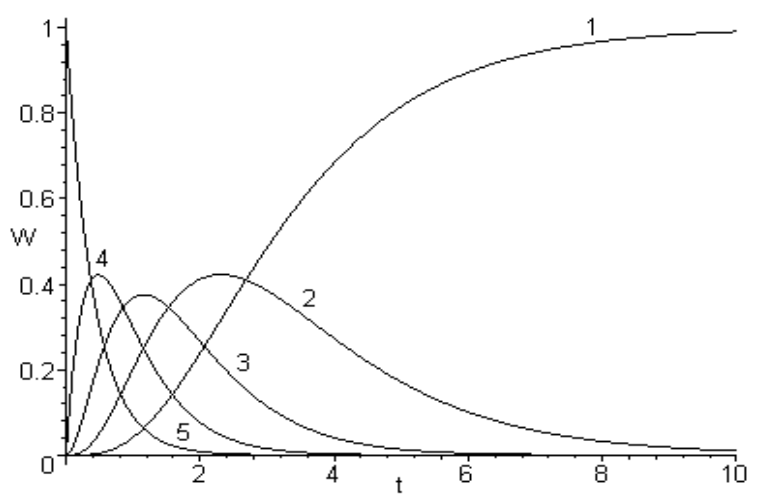

FIG. 2: W-functions versus time for pure aggregation process. $G=5, \gamma=1$. All curves $W(s, t)$ are marked by the number $s$ of the proper state.

\section{SIMILAR FRAGMENTATION PROCESS}

Let us consider a process of pure fragmentation (dissociation, decay) of clusters. We assume the inner $G$-cluster structure at $t=0$ to be similar to the picture at $t=0$ with $G$ cars of unit mass in Sect. II and, thus, with $G-1$ intervals between them . An analogous assumption applies to all the $s$-clusters at $t \neq 0$. We will understand "the similarity of an inner cluster structure and an outer one" in such a purport. In addition, let us suppose a qualitative equivalence of all the inner intervals between constituents of a cluster.

At the above pure aggregation mechanism the place where a coalescence act has happened is partly forgotten. When two adjacent clusters enumerated $k_{s}$ and $(k+1)_{s}$ according to enumeration of an $s$-cluster state with sizes (see below) $\left(g \prime_{k}\right)_{s}$ and $\left(g \prime_{k+1}\right)_{s}$ coagulate, a new cluster of the size $\left(g_{k}\right)_{s-1}=\left(g \prime_{k}\right)_{s}+\left(g_{k+1}\right)_{s}$ arises. The cluster ordinal number $k_{s-1}$ is given in terms of the new $(s-1)$ cluster state which originates from the act of coagulation as in Sect. III.

Evidently, a partial loss of memory on the way in which the microscopic state has been created comes about at the very place because the inverse problem of one-toone rebuilding of the previous $s$-cluster state (the outer state) cannot be solved. On the other hand, the things are the same with the interior structure of some cluster. Its cluster parents cannot be reconstructed one-to-one as well. When one looks at a system state or some cluster as something given, the exact information about ordinal numbers and sizes of adjacent cluster parents and even the mother state is forgotten. That is why one can talk about a loss of memory in such a process. In the whole it is the reason to postulate some equivalence of constituents and intervals between them in the inner cluster space.

This set of assumptions results in the dependence of the probability functions on the size of a cluster and the time only.

Let us realize the cluster size as the number of particles confined into the given cluster, let $\gamma$ be the rate of an elementary fragmentation act.

If a fragmentation rate is proportional to the cluster size minus unit, i.e., the number of possible rupture 
places is equal to the number of inner intervals, the equation

$$
\frac{d W(s, t)}{d t}=\gamma[(G-s+1) W(s-1, t)-(G-s) W(s, t)],
$$

with initial conditions

$$
W(1,0)=1 ; \quad W(s, 0)=0, \quad \text { if } s \neq 1,
$$

describes the process under consideration. The r.h.s. of Eq. (26) consists of a gain term due to decay of clusters belonging to an $s-1$-cluster state and a loss term due to decays of those clusters belonging to $s$-cluster state that produces the clusters pertaining to an $s+1$-cluster state. Equation (26) can be solved by using the generating function introduced by the equation

$$
\begin{aligned}
& \frac{\partial}{\partial t} \mathrm{~F}(z, t)-\left(-z^{2} \gamma+z \gamma\right) \frac{\partial}{\partial z} \mathrm{~F}(z, t) \\
& \quad=\gamma(z G-z-G+1) \mathrm{F}(z, t)
\end{aligned}
$$

with the solution

$$
F(z, t)=-\frac{\left(z+e^{(-\gamma t)}-e^{(-\gamma t)} z\right)^{G}}{-z-e^{(-\gamma t)}+e^{(-\gamma t)} z} .
$$

Of course, one recognizes a usual Poissonian process here. It seems contextual and, hence, quite reasonable to name such a process as a similar fragmentation (process).

For example, for $G=5$

$$
\begin{gathered}
\mathrm{W}_{1}=e^{(-4 \gamma t)} \\
\mathrm{W}_{2}=4 e^{(-3 \gamma t)}-4 e^{(-4 \gamma t)} \\
\mathrm{W}_{3}=6 e^{(-2 \gamma t)}-12 e^{(-3 \gamma t)}+6 e^{(-4 \gamma t)} \\
\mathrm{W}_{4}=4 e^{(-\gamma t)}-12 e^{(-2 \gamma t)}+12 e^{(-3 \gamma t)}-4 e^{(-4 \gamma t)} \\
\mathrm{W}_{5}=1-4 e^{(-\gamma t)}+6 e^{(-2 \gamma t)}-4 e^{(-3 \gamma t)}+e^{(-4 \gamma t)}
\end{gathered}
$$

The average number of clusters reads

$$
\bar{s}(t)=\left.\partial_{z} z F(z, t)\right|_{z=1}=e^{(-\gamma t)}+G\left(1-e^{(-\gamma t)}\right) .
$$

\section{PROCESS OF AGGREGATION AND SIMILAR FRAGMENTATION}

Let us consider such a clustering process, which runs as a result of some combined action both of the aggregation and the similar fragmentation. Let $\gamma_{1}$ and $\gamma_{2}$ be constant rates of an elementary coalescence act and an elementary fragmentation act, respectively,

$$
\begin{gathered}
\frac{d W(s, t)}{d t} \\
=\gamma_{1}[s W(s+1, t)-(s-1) W(s, t)] \\
-\gamma_{2}[(G-s) W(s, t)-(G-s+1) W(s-1, t)],
\end{gathered}
$$

with initial conditions

$$
W(G, 0)=1 ; \quad W(s, 0)=0, \quad \text { if } s \neq G .
$$

The r.h.s. of Eq. (36) consists of gain terms due to coagulation of clusters from an $(s+1)$-cluster state and dissociation of those clusters belonging to an $(s-1)$-cluster state and loss terms due to simultaneous coalescence and dissociation of clusters belonging to an $s$-cluster state. To make things more clear, we could rewrite Eq. (36) in the form

$$
\begin{gathered}
\frac{d W(s, t)}{d t} \\
=\left(\gamma_{1} s W(s+1, t)+\gamma_{2}(G-s+1) W(s-1, t)\right) \\
-\left(\gamma_{1}(s-1) W(s, t)+\gamma_{2}(G-s) W(s, t)\right) .
\end{gathered}
$$

These equations can be solved by using the generating function $F(z, t)$ defined by the equation

$$
\begin{gathered}
\frac{\partial}{\partial t} \mathrm{~F}(z, t) \\
-\left(-z^{2} \gamma_{2}-z \gamma_{1}+z \gamma_{2}+\gamma_{1}\right) \frac{\partial}{\partial z} \mathrm{~F}(z, t) \\
=\gamma_{2}(z G-z-G+1) \mathrm{F}(z, t),
\end{gathered}
$$

whose solution is

$$
\begin{gathered}
F(z, t) \\
=\gamma\left(\frac{z \gamma_{2}+\gamma_{1} e^{(-t \gamma)} z-\gamma_{1} e^{(-t \gamma)}+\gamma_{1}}{\gamma}\right)^{G} / \\
\left(z \gamma_{2}+\gamma_{1} e^{(-t \gamma)} z-\gamma_{1} e^{(-t \gamma)}+\gamma_{1}\right),
\end{gathered}
$$

where

$$
\gamma=\gamma_{1}+\gamma_{2}
$$

For example, for $G=5$

$$
\mathrm{W}_{1}=.6830134554\left(e^{(-1.1 t)}-1\right)^{4}
$$

$$
\mathrm{W}_{2}=-2.732053822\left(e^{(-1.1 t)}-1\right)^{3}\left(.1+e^{(-1.1 t)}\right),
$$

$$
\mathrm{W}_{3}=4.098080732\left(e^{(-1.1 t)}-1\right)^{2}\left(.1+e^{(-1.1 t)}\right)^{2}
$$

$$
\mathrm{W}_{4}=-2.732053822\left(e^{(-1.1 t)}-1\right)\left(.1+e^{(-1.1 t)}\right)^{3} \text {, }
$$

$$
\mathrm{W}_{5}=.6830134554\left(.1+e^{(-1.1 t)}\right)^{4} \text {. }
$$

The average number of clusters is given by the formula

$$
\begin{gathered}
\bar{s}(t)=\left.\partial_{z} z F(z, t)\right|_{z=1} \\
=1-\frac{\gamma_{2}+\gamma_{1} e^{(-t \gamma)}}{\gamma}+\frac{G\left(\gamma_{2}+\gamma_{1} e^{(-t \gamma)}\right)}{\gamma} .
\end{gathered}
$$




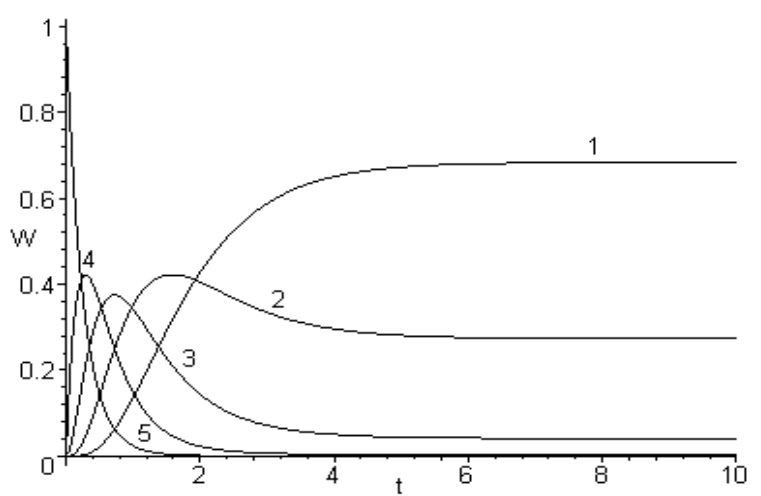

FIG. 3: W-functions. Process of aggregation and similar fragmentation. $G=5, \gamma=1.5, \gamma_{1}=1, \gamma_{2}=0.5$. All curves $W(s, t)$ are marked by the number $s$ of the proper state.

\section{PROCESS WITH AN ARBITRARY FRAGMENTATION}

Let us assume that clusters lose the memory of their aggregation history completely. This means that a hypothetical mechanism of such a kind aggregation loses not only the place of a coalescence act (Sect. VI), but in this case the inner structure of a cluster does not coincide with outer one, becomes a uniform one, and let us say "is beside itself". Shortly speaking, the mass distribution inside a cluster becomes a uniform one under such an aggregation, i.e., the inner structure does not depend on the cluster size (see Sect. VI, and hence, the actual number of inner intervals (see Sect. VII) is equal to 0.

However, let us apply the "three-level bunch" scheme of transitions (connections) between the three nearest cluster-states, widely used above. That is, in fact, one of the main subjects under consideration in this paper.

In this case, it is reasonable to assume that the fragmentation rate depends solely on the outer cluster state indices. I.e., the rate depends on the integer number of the clusters or the intervals minus unit between them for the states belonging to such a three-level bunch. Adding the contextual "natural" condition of stopping the fragmentation process at $s=G$, the corresponding equation reads

$$
\begin{aligned}
& \frac{d W(s, t)}{d t}=\gamma_{1}[s W(s+1, t)-(s-1) W(s, t)]-\gamma_{2} \\
& \times[(1-\Delta(G-s)) s W(s, t)-(s-1) W(s-1, t)],
\end{aligned}
$$

with initial conditions

$$
W(G, 0)=1 ; \quad W(s, 0)=0, \quad \text { if } s \neq G,
$$

where $\gamma_{1}$ and $\gamma_{2}$ are the constant rates of an elementary coalescence act and of an elementary fragmentation act, respectively. Equation (48) is constructed so as to absorb the conditions $W(G+1, t \geq 0)=0, W(0, t \geq 0)=0$. We shall see below, the solutions of Eq. (48) bear out this assertion.
It should be noted, that the mass spectra of clusters for process Eqs. (48) and (49) differ from those in Sect. III, V. $\mathrm{G}=5$,

Equation (48) can be solved for each $G$. If, for example,

$$
\begin{gathered}
W(s, t)=\sum_{i=1}^{G} C_{i} e^{\lambda_{i} t} A_{s i}, \\
C=[.44721,-.44721,-.44721,-.44721,-.44721], \\
\lambda=[-10.95389,-5.73117,-2.57163,-.74329,0], \\
A=\left[\begin{array}{c}
.01096, .11376,-.43096, .77537,-.44721 \\
-.10909,-.53825, .67732, .19904,-.44721 \\
.42839, .67814, .36055,-.16309,-.44721 \\
-.77746, .19355,-.15969,-.36411,-.44721 \\
.44721,-.44721,-.44721,-.44721,-.44721
\end{array}\right] .
\end{gathered}
$$

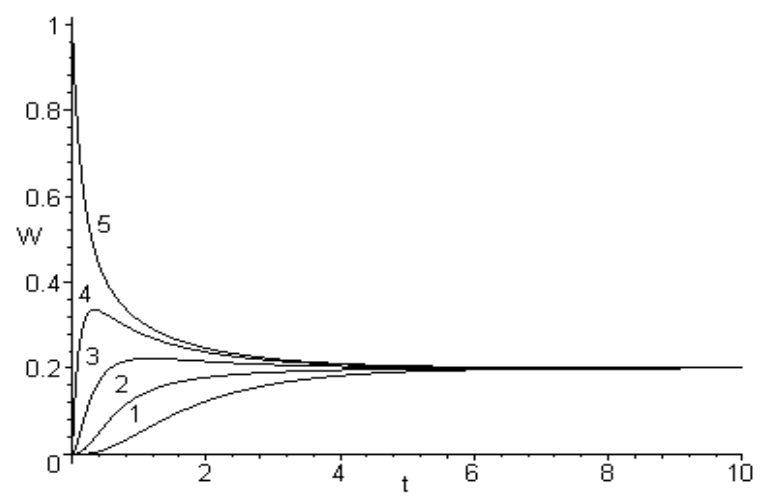

FIG. 4: W-functions. Aggregation with an arbitrary fragmentation. $G=5, \gamma=2, \gamma_{1}=1, \gamma_{2}=1$. All curves $W(s, t)$ are marked by the number $s$ of the proper state.

\section{CONCLUSION}

In this paper, we are interested in kinetics of cluster formation and construct a number of analytically solved models of such a process. It does not matter whether a motor lane, a computational network, space dust or glass is a real environment to match such a script. The illustration of the problem in terms of a traffic jam is not a specific one. The known technical and natural phenomena of traffic jam or aggregation (coagulation) and their evolution have been considered as processes of clustering (nucleation).

Brought forward in this work, the kinetics models take account of both the aggregation (coagulation) and the fragmentation (decay) processes. Previous studies of nonlinear Lotka-Volterra systems [7] brought us to a search for a possibility to give a linear description of those very complicated and nonlinear situations or for something akin to such a picture. A dynamical description of some system could be substituted by a stochastic 
one. We consider one-dimensional cases (in the coordinate sense) but the scenarios should be valid for the three dimensions if there is a spherical symmetry.

When we deal with a system of a finite number of particles, a natural way of the above substitution is to use the language of enumerated states of that system. A state of that sort is characterized by the population number and probability function to reveal the system itself in this state exactly. The "three-level bunch" scheme of transitions between the three nearest states of the system is natural as well. Of course, that probability should depend upon the probability of something else to happen. In the case considered, it could be an act of coalescence or decay ( dissociation, fragmentation, etc., where the term depends on the application ). Thus, we have just met a product of probabilities. What can one do?

The probability (rate) of the above acts could be dependent or independent on the system states or its particular attributes. In the case of such a dependence, one can say nothing without a special investigation. On the contrary, the rate independence of the above circumstances makes the situation a linear one in the state probability function. Let us take into account that the rates may be independent not only of macro parameters but also of the cluster size (e.g., if coalescence/fragmentation depends only on the valency of some chemical clusters). This leads to our key mathematical assumption of the above rates being constant. That is why we obtain linear analytically solved master equations of that clustering kinetics, which are also evolutionary-type equations.

The very method to solve most of those problems is in use of the generating function.

But some peculiarities of applying of this method for solving the linear differential-difference equations are revealed. It becomes useless when the structure homogeneity of terms in the r.h.s. of equations discussed is violated as a result of combining aggregation and fragmentation terms, i.e., owing to violation of the Markovian semigroup structure of those r.h.s. The latter, as well as symmetry, momenta and other questions (e.g. universality of the three-level bunch scheme ) not included in this sketch will be considered in more detail in papers to follow, as well as some details of the utilization of a MAPLE-program, used to obtain some analytical results, which is to be published in 9].

Other questions about the mass spectra and the singlecluster mass distribution in a pure aggregation process help to display the combinatorial nature of the problem under consideration and its closeness to some population dynamics problem. They are solved by means of the Laplace transformation, which leads to the calculation of certain convolutions. The properties of those convolutions are true due to the isomorphism between a set of generating functions with a product operation and a set of $w(g)$ with a convolutions, which results in a useful formula.

It should be remembered, that we considered two types of fragmentation: the similar fragmentation and the ar- bitrary one. They lead to different results. Such a difference can be realized as a manifestation of distinction between collective and more discrete additive properties of a cluster on a very abstract level, far off a specific nature of that cluster. Moreover, we shall see the possible existence of several aggregation mechanisms (for a variety of reasons). Thus, one and the same stochastic description of the aggregation can mask a number of processes diverse in their dynamical details.

However, the generality of the results is restricted. The conjecture that the rates are constant $\left(\gamma_{1,2}=\right.$ const $\left._{1,2}\right)$ is nowadays somewhat at odds with what is known about molecular and atomic clusters, and processes for nanoscale units of matter. Obviously, the mentioned conjecture reduces a mathematical universality as well. We are grateful to the referee for drawing our attention to the necessity of making this remark.

Nevertheless, the authors hope that appropriate processes of such a type may be found by the following reason. The presented formalism is independent of any physical scale. The only conjecture of the possibility to draw distinction between certain particles and intervals between them has been done. Such an admissibility could be combined with not only a classical conception but a quantum one under some conditions (e.g., if the first Born approximation is valid ) as well.

Present work belongs to the stream originated from the famous Smoluchovski's articles [8].

\section{Acknowledgments}

The authors are very grateful to V.B. Priezzhev for discussions and numerous kind advice.

\section{APPENDIX A}

Let

$$
\begin{gathered}
N_{0}=0,1,2, \ldots, \\
a(n)=a_{n}, n \in N_{0}, \\
A=\left\{a: N_{0} \rightarrow R\right\}, \\
F=\left\{f: C \rightarrow C, f(z)=\sum_{n \in N_{0}} a_{n} z^{n}\right\} .
\end{gathered}
$$

The reversible mapping $Z: A \rightarrow F$

$$
f=Z(a) \text { so as } f(z)=\sum_{n \in N_{0}} a(n) z^{n}
$$

has the inverse mapping $Z^{-1}$

$$
\begin{gathered}
a=Z^{-1}(f) \text { so as } a(n)=\oint f(z) \frac{d z}{z^{n+1}}, \\
n \in N_{0} .
\end{gathered}
$$


The convolution $\circ$ is an operation on a set A such that

$$
c=a \circ b \quad \text { iff } \quad c(k)=\sum_{j=0}^{k} a(k-j) b(j) .
$$

The mapping $\mathrm{Z}$ is a morphism of a semigroup $(A, \circ)$ to a semigroup $(F, \cdot)$. Z maps the convolution $\circ$ to the product · :

$$
c=a \circ b \rightarrow Z(c)=Z(a) Z(b)
$$

The associative and commutative semigroup $(A, \circ)$ has the unit $\Delta$ :

$$
\begin{gathered}
\Delta(0)=1, \Delta(n)=0, n \neq 0, \\
Z(\Delta)=1, Z^{-1}(1)=\Delta .
\end{gathered}
$$

Here is a useful formula

$$
=\sum_{\left\{j_{k} \in N_{0}\right\}} \begin{gathered}
\left(a_{1} \circ a_{2} \circ \ldots \circ a_{n}\right)(m) \\
a_{1}\left(j_{1}\right) \ldots a_{n}\left(j_{n}\right) \Delta\left(m-j_{1}-\ldots-j_{n}\right)
\end{gathered}
$$

[1] V.M. Dubovik, A.G. Galperin, A.A. Lushnikov, V.S. Richvitsky, Communication of JINR, (E5-2000-215, Dubna, 2000); in Second Intern. Conf. MTCP, July 2429, 2000, p.62, (JINR, Dubna, Russia.)

[2] Nucleation Theory and Applications, 10th Anniversary, edited by J.W.P. Schmelzer, G. Roepke, V.B. Priezzhev, (JINR, Dubna, 1999).

[3] V.S. Safronov. Evolution of the Pre-Planetary Cloud and the Formation of the Earth and Planets, (Msc., Nauka, p.244) (Engl.transl.1971 Israel program for scientific translations)

[4] The Ecology of Computation, edited by B.A. Huberman, (Amster.- NY - Oxford-Tokyo, 1988).

[5] H. Haken, Synergetics, (Springer-Verlag, BerlinHeidelberg-NY, 1978).

[6] G. Nicolis, I. Prigogine, Exploring Complexity, (Freeman, San-Francisko, 1989).

[7] V.M. Dubovik, A.G. Galperin, V.S. Richvitsky, Non-
lin.Proc.Compl.Sys., v3, N4 (2000); V.M. Dubovik, A.G. Galperin, V.S. Richvitsky, S.K. Slepnyov, from 'Yad.Fiz' v.63, N4, 695-700 (2000) [Phys.Atom.Nucl, v.63, N4, 629-634 (2000)] V.S. Richvitsky, A.G. Galperin, V.M. Dubovik.(Preprint JINR P4-98-42,Dubna, 1998) - in Russian, in cyrillic: ..., .., .. ( 4-98-42, ,1998); A.G. Galperin, V.M. Dubovik, V.S. Richvitsky.(Preprint JINR P4-97340,Dubna, 1997) - in Russian, in cyrillic: A. ., . ., . . ( P4-97-340, , 1997).

[8] M. von Smoluchovski, Physik.Zeitshrift, v.17, 557-585 (1916); M. von Smoluchovski, Z.Phys.Chem., v.92, 124 (1917).

[9] V.M. Dubovik, A.G. Galperin, V.S. Richvitsky, Lushnikov A.A. Solution of certain equations of cluster growth and fragmentation kinetics in Maple package, JCMSE (the Journal of Computational Methods in Sciences and Engineering), (to be published in March-April 2002). 